\title{
Inferior oblique muscle of the eye: its foetal development with special reference to understanding of the frequent variants in adults
}

\author{
Z.W. Jin'10, S. Umeki², Y. Takeuchi², M. Yamamoto²®, G. Murakami², 3(D, \\ S. Abe ${ }^{2}$, J.F. Rodríguez-Vázquez ${ }^{4}(\mathbb{B}$ \\ 'Department of Anatomy, Wuxi School of Medicine, Jiangnan University, Wuxi, China \\ ${ }^{2}$ Department of Anatomy, Tokyo Dental College, Tokyo, Japan \\ ${ }^{3}$ Division of Internal Medicine, Cupid Clinic, Iwamizawa, Japan \\ ${ }^{4}$ Department of Anatomy and Embryology, School of Medicine, Complutense University, Madrid, Spain \\ [Received: 13 March 2021; Accepted: 9 April 2021; Early publication date: 28 April 2021]
}

\begin{abstract}
Background and Materials and methods: To provide better understanding of frequent variations of the inferior oblique (IO) of adult extraocular muscles, we observed sagittal and horizontal histological sections of the eye and orbits from 32 foetuses (7-34 weeks of gestational age; 24-295 mm of crown-rump length). Results: In early foetuses (7-8 weeks), the 10 was restricted at an antero-infero-medial angle of the future orbit. In contrast to extraocular recti, the 10 appeared to extend along the mediolateral axis and had no definite tendon. At midterm, the $1 O$ tendon became evident. Sometimes, the 10 muscle belly attached to the inferior rectus or, the 10 tendon divided into two laminae to enclose the lateral rectus. At late-term, a multilayered sheath was evident around the sclera and, via one or some of the fascial layers, the 10 was communicated with a fascia enclosing the inferior rectus. At midterm and late-term, the 10 originated not only from the maxilla near the orbicularis oculi origin but also from a vein-rich fibrous tissue around the lacrimal sac. Both origins were muscular without intermittent tendon or ligament. Therefore, the fascial connection as well as a direct contact between the $I O$ and the inferior or lateral rectus seemed to provide variant muscular bridges as reported in adults. Moreover, the two attachment sites at the origin seemed to provide double muscle bellies of the adult 10.

Conclusions: Consequently, the present specimens contained seeds of any types of adult variations. The muscle fibres from the lacrimal sac might play a role for the lacrimal drainage. (Folia Morphol 2022; 81, 2: 442-450)
\end{abstract}

Key words: inferior oblique muscle of the eye, inferior rectus, lateral rectus, human foetus, muscular bridge, double muscle bellies

\section{INTRODUCTION}

The inferior oblique muscle (IO) shows anatomic variations most frequently in extraocular muscles [8].
The 10 is usually or often composed of double or multiple muscle bellies: $8 \%$ in DeAngelis and Kraft [1]; $17 \%$ in DeAngelis et al. [2]; more than $90 \%$ in Yalçin

Address for correspondence: Z.W. Jin, MD, PhD, Department of Anatomy, Wuxi School of Medicine, Jiangnan University, 1800 Lihu Avenue, Wuxi, Jiangsu, 214122, China, tel: +86-510-8519-7079, fax: +86-510-8519-3570, e-mail: zwjin@jiangnan.edu.cn

This article is available in open access under Creative Common Attribution-Non-Commercial-No Derivatives 4.0 International (CC BY-NC-ND 4.0) license, allowing to download articles and share them with others as long as they credit the authors and the publisher, but without permission to change them in any way or use them commercially. 
and Ozan [14]. A muscular bridge was also reported between the 10 and inferior rectus: $6.6 \%$ in Yalçin et al. [13]. The obliquus accessorius inferior is also known originating from the orbital apex and inserting to a site adjacent to the inferior rectus insertion [8] although it is similar to the inferior rectus rather than the IO. In contrast to the usual close relation with the inferior rectus at the insertion, two case reports demonstrated the 10 insertion near or adjacent to the lateral rectus insertion [8]. The first aim of this study was to provide better understanding as to why and how the frequent variants occur in foetal development of the IO.

Another interest of the foetal 10 was the muscle belly wrapping around the eyeball: the morphology is quite different from the superior oblique muscle in which a cartilaginous pulley, the trochlea, makes the acute turn of the tendon. Our group hypothesized that the superior oblique tendon is "secondarily" attached to and turned by the trochlea due to rotation of the foetal eyeball [7]. Harayama et al. [6], on the basis of detailed morphometrical data, concluded that medial rotation of the eye ball occurs at 12 weeks and at a stage between 16 and 19 weeks of gestation. De Haan et al. [3] also described the rotation in the prenatal stage. The second aim of this study was to describe a developmental process to form the curved IO belly extending along the eyeball. In contrast to the four recti and levator palpebrae superioris, previous descriptions on the foetal 10 were very limited [12].

\section{MATERIALS AND METHODS}

This study was conducted in accordance with the Declaration of Helsinki 1995 (as revised in 2013). We examined paraffin-embedded histological sections from 32 foetuses (7-34 weeks of gestational age [GA]; 24-295 mm of crown-rump length [CRL]). The specimens were categorised into three groups according to age and size: 1) 7 early foetuses at GA 7-8 weeks (CRL 24-35 mm; O'Rahilly's stage 21-23); 2) 18 midterm foetuses at GA 12-15 weeks (CRL 71$-115 \mathrm{~mm}$ ); 4) 7 late-term foetuses at GA 25-34 weeks (CRL 200-295 mm). All sections were part of the large collection kept at the Department of Anatomy of the Universidad Complutense, Madrid, and were the results of miscarriages and ectopic pregnancies from the Department of Obstetrics of the University. Sections of the embryos, early foetuses and midterm foetuses had been prepared serially, while the late- stage foetuses had been sectioned 50 or 100 micron interval. The sections were stained with haematoxylin and eosin (HE) or Azan. The sectional plane was often sagittal (26 specimens) and sometimes horizontal (4 of midterm foetuses; 2 of late-term foetuses). This study was approved by the Ethics Committee of Complutense University (B08/374). Most photographs were taken with a Nikon Eclipse 80.

\section{RESULTS}

\section{Observations of sagittal sections from 7 early foetuses}

At GA 7 weeks (Fig. 1), the 10 was restricted at a small area near the medial angle of the conjunctiva: this site corresponded to an antero-infero-medial angle of the future orbit. In contrast to the extraocular rectus muscles along the long axis of the developing eyeball, the 10 extended along the medio-lateral axis. Thus, in sagittal sections, the 10 was cut transversely, while the rectus longitudinally (Fig. 1D, H). The IO was located outside of and inferior to the inferior rectus.

At GA 8 weeks (Fig. 2), the extraocular recti had a distinct tendon to merge with the dense, anterior part of the sclera (Fig. 2C). In contrast, the IO carried no definite tendon and the posterior end dispersed into a loose, posterior part of the sclera (Fig. 2E). The posterior part of the 10 was close to the lateral rectus muscle belly (Fig. 2E). The 10 muscle belly crossed obliquely in the lateral side of the inferior rectus and extended posterosuperiorly to wrap around the eyeball (Fig. 2B, F). Thus, the IO obtained the final curved course along the eyeball as earlier as the extraocular rect. No cartilage or membranous bone appeared at an antero-infero-medial angle of the future orbit. The orbicularis oculi muscle was separated from the 10 origin by a loose mesenchymal tissue (Fig. 2A). Likewise, no hard tissue separated the IO from the infraorbital nerve (Fig. 2G). The IO origin still took a tendon-like appearance at the anterior end (Fig. 2A).

\section{Observations of sagittal sections from 14 midterm foetuses}

At GA 12-15 weeks, the IO originated from the maxilla near the origin of the pars orbitalis of the orbicularis oculi muscle and both muscle origins sandwiched a dense fibrous tissue (Fig. 3). The 10 origin was muscular: thus, each muscle fibres appeared to attach to the fibrous tissue. The IO tendon merged with 

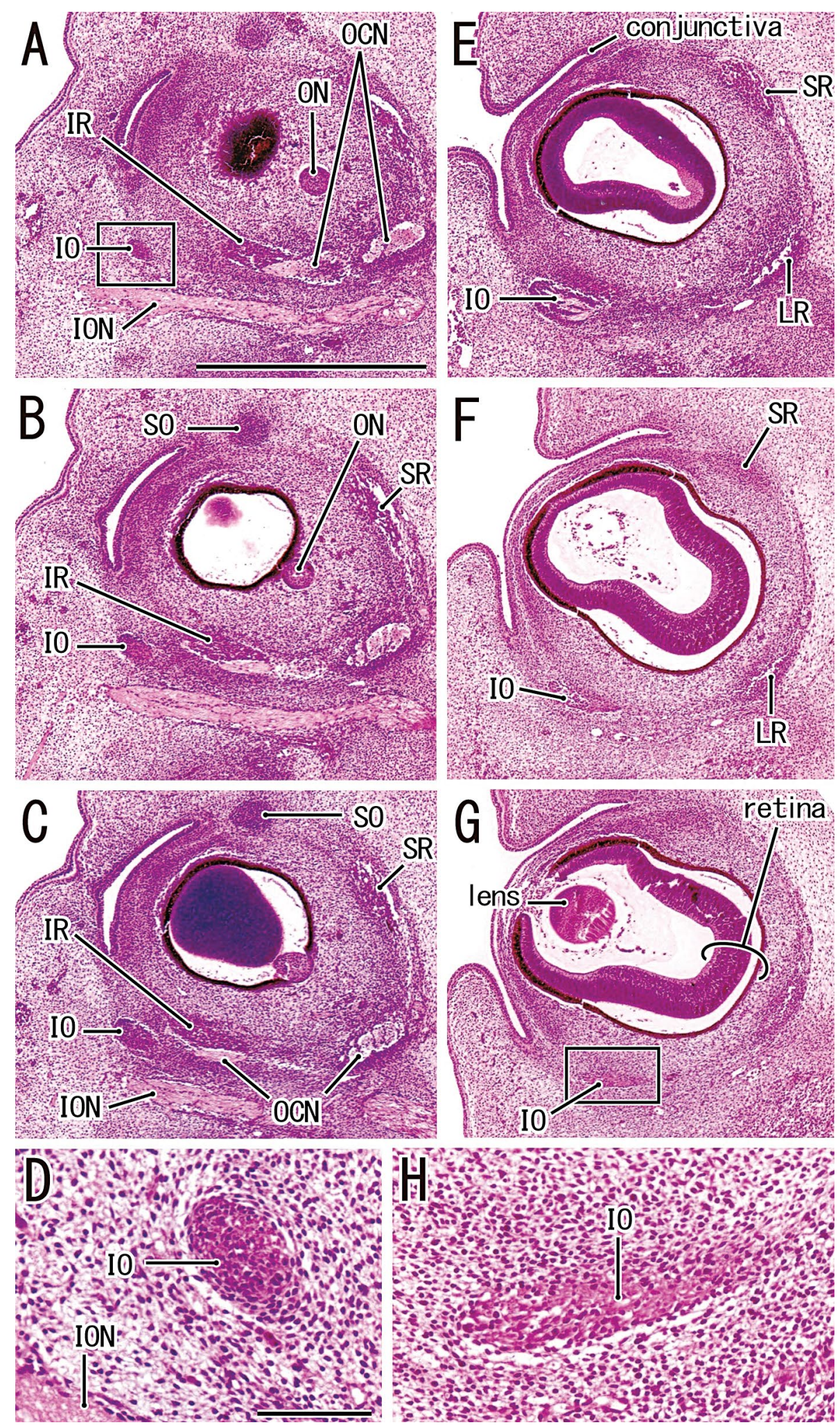

Figure 1. A-H. Inferior obliquus muscle in an embryo of $24 \mathrm{~mm}$ crown-rump length. Sagittal sections. Haematoxylin and eosin staining. Panel A displays the most medial plane in the figure. Panels $D$ and $H$ are higher magnification views of squares in panels $A$ and $G$, respectively. A future muscle origin of the inferior obliquus $(I O)$ is a dense mesenchymal condensation near the infraorbital nerve (ION), but no cartilaginous tissue is developed at the origin (panels $A$ and $D$ ). A future insertion of the $I 0$ is identified as a primitive tendon-like band in the inferior side of the eyeball (panels $G$ and $H$ ). Panels $A-C$ and $E-G$ (or panels $D$ and $H$ ) were prepared at the same magnification. Scale bars: $1 \mathrm{~mm}$ in panel $A$ and $0.1 \mathrm{~mm}$ in panel $\mathrm{D} ; \mathrm{IR}$ — inferior rectus; 10 — inferior obliquus; ION — infraorbital nerve; LB — lacrimal bone; $L R$ — lateral rectus; $L S$ lacrimal sac; $\mathrm{MX}$ - maxilla; NLD — nasolacrimal duct; $\mathrm{OCN}$ - oculomotor nerve; $\mathrm{ON}$ — optic nerve; 00 — orbicularis oculi; $\mathrm{SO}$ - superior obliquus; SR - superior rectus. 

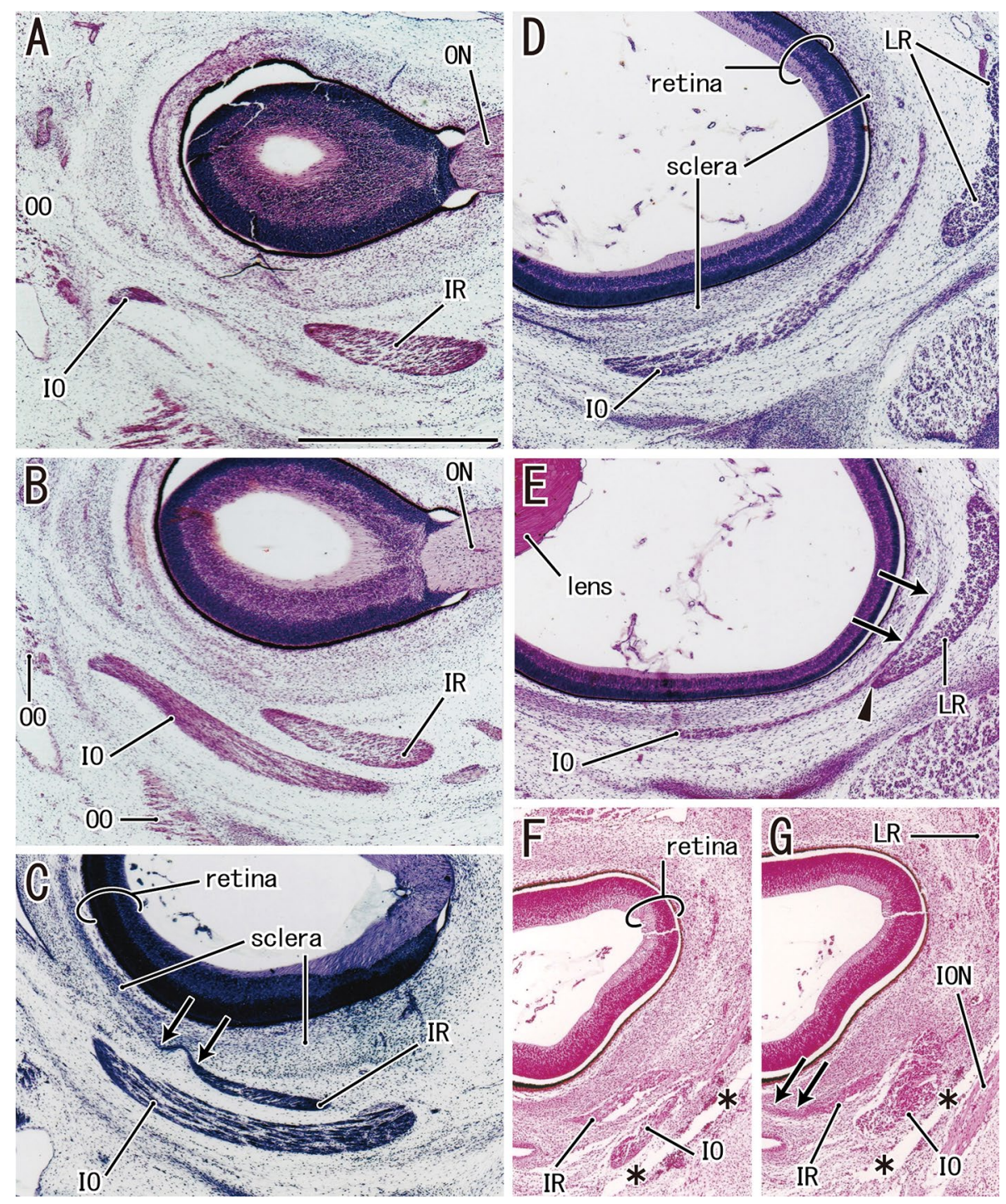

Figure 2. A-G. Inferior obliquus muscle in early foetuses of 35 and $38 \mathrm{~mm}$ crown-rump length, respectively. Haematoxylin and eosin staining (panels $A, B, D-G$ ) or Azan staining (panel C). Panels A-E display sagittal sections from a foetus of $38 \mathrm{~mm}$, while panels $F$ and $G$ a foetus of $35 \mathrm{~mm}$. Panel A displays the most medial plane in the former specimen. Origins of the inferior obliquus $(I 0)$ and orbicularis oculi $(00)$ sandwich a loose mesenchymal tissue (panel A). A tendinous insertion of the inferior rectus (IR) merges with a dense tissue of the sclera anterior part (arrows in panel C), while that of the inferior obliquus disperses in a loose tissue of the sclera posterior part (arrows in panel E). Near the insertion, the inferior obliquus and lateral rectus (LR) are closely located (arrowhead in panel E). Panels $F$ and $G$ exhibit a crossing between the inferior rectus and obliquus muscles. Arrows in panel $G$ indicate an initial tendon of the inferior rectus. Asterisks in panels $F$ and $G$ indicate an artefact space produced during histological procedure. All panels were prepared at the same magnification (scale bar in panel A: $1 \mathrm{~mm}$ ). Other abbreviation — see Figure 1.

the posterior part of the sclera (Fig. 3D, E), while the inferior rectus inserted to the anterior part (Fig. 3C). The sclera appeared to be dense and homogeneous in the anterior part, while the posterior part was still composed of a relatively loose bundle of irregular fibres. We found two types of the 10 variations: 1) the IO muscle belly attached to the inferior rectus in 1 specimen (Fig. 3A) and 2) the 10 tendon divided into two laminae to enclose the lateral rectus in two specimens (Fig. 3F).

\section{Observations of sagittal sections from 5 late-term foetuses}

A multilayered sheath or fascia was much or less evident around the sclera (Figs. 4, 5): a thick fascia from the conjunctiva was particularly thick and it extended posteriorly to disperse into: 1) the orbital fat (Figs. 4B, 5B), 2) a fascia covering the IO (Fig. 4D) and/or 3) a fascia enclosing the inferior rectus (Fig. 5C). The fascia covering the 10 was communicated with the other fascia enclosing the inferior rectus 


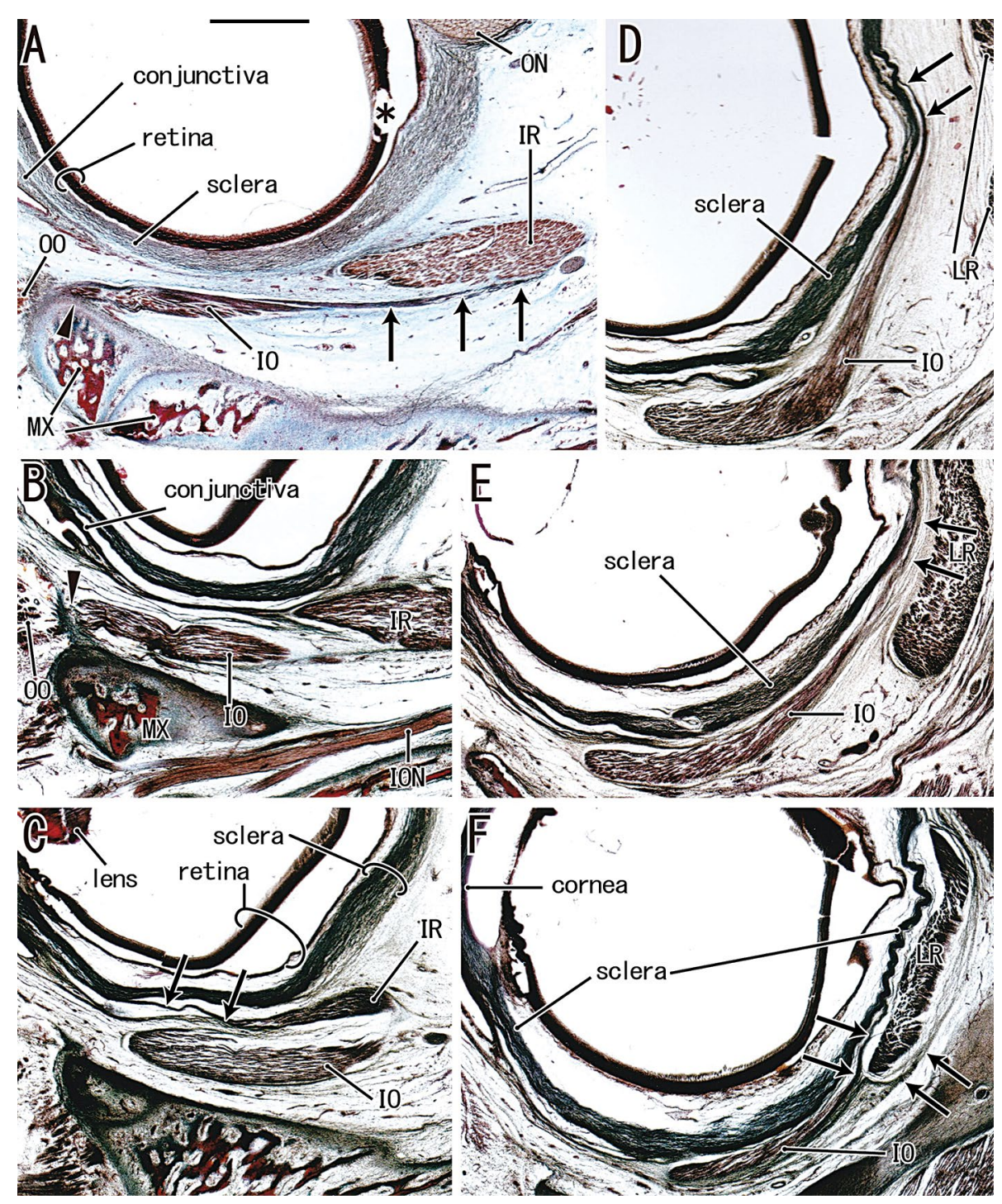

Figure 3. A-F. Inferior obliquus muscle in two midterm foetuses of 92 and $97 \mathrm{~mm}$ crown-rump length, respectively. Panels $\mathrm{A}$ (Azan staining) displays a sagittal section from a foetus of $92 \mathrm{~mm}$, while panels B-F (Azan but long years after staining) a foetus of $97 \mathrm{~mm}$. Panel B displays the most medial plane in the latter specimen. Origins of the inferior obliquus (I0; arrowhead) and orbicularis oculi (00) are muscular and they sandwiched a band-like tissue attaching to the maxilla (MX; panels $A$ and $B$ ). In the former foetus, the inferior obliquus appears to attach to the inferior rectus (arrows in panel A). In the latter foetus, a tendinous insertion of the inferior obliquus not only joins the sclera (arrows in panels $D$ and $E$ ) but divides into two laminae to enclose the lateral rectus ( $L R$; arrows in panel F). The inferior rectus (IR) makes a tendinous insertion to the sclera (arrows in panel C). Asterisk in panel A indicates an artifact space produced during histological procedure. All panels were prepared at the same magnification (scale bar in panel A: $1 \mathrm{~mm}$ ). Other abbreviation — see Figure 1.

(Fig. 4A, 5C). The sclera was composed of regularly-arrayed fibres (Fig. 4F, 5G). The 10 tendon merged with the most external layer of the sclera (Figs. 4F, 5G). At the origin from the maxilla, the $\mathrm{IO}$ and orbicularis oculi sandwiched a dense fibrous tissue (Fig. 5A). Because of no connection to either the tarsal plate or lacrimal sac, this tissue did not correspond to a part of the medial palpebral ligament. The orbicularis oculi originated not only from the maxilla but from the lacrimal bone (Fig. 5C, D).

\section{Observations of horizontal sections from 4 midterm and 2 late-term foetuses}

Horizontal sections well demonstrated an entire area of the 10 covering the eyeball as well as an almost anteroposterior direction of the muscle fibres (Fig. 6). However, sagittal sections were better useful than horizontal sections for demonstrations of the curved muscle belly wrapping around the eyeball. Notably, all of the six specimens for horizontal sections carried two muscle attachments at the antero-infero-medial 


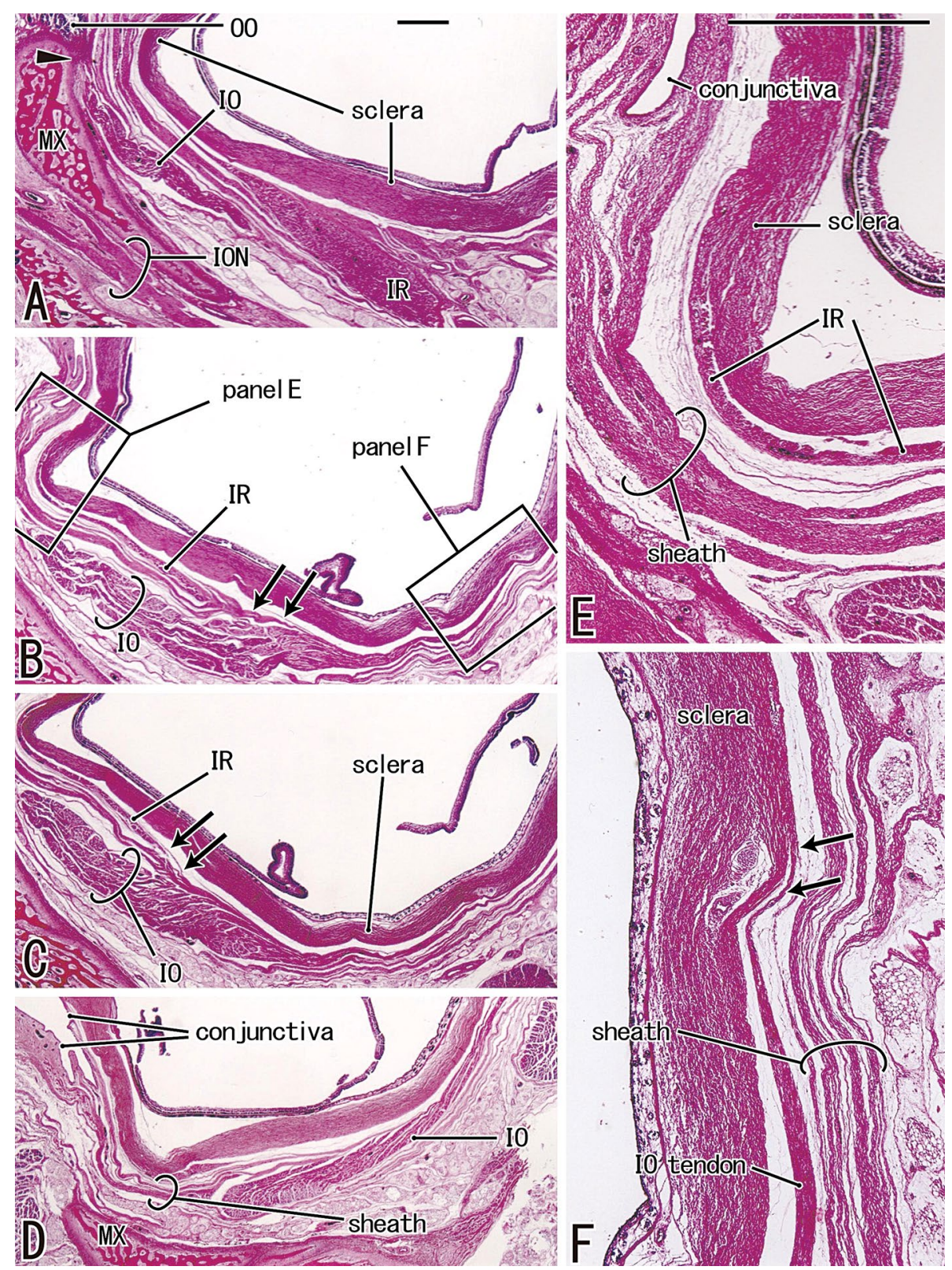

Figure 4. A-F. Inferior obliquus muscle in a late-term foetus of $270 \mathrm{~mm}$ crown-rump length. Haematoxylin and eosin staining. Sagittal sections. Panel $A$ displays the most medial plane in the figure. Panels $E$ and $F$ are higher magnification views of squares in panel $B$. Panel $A$ shows a muscular origin of the inferior obliquus (I0; arrowhead). The inferior rectus (IR) connects with the inferior obliquus by a fascia (arrows in panels $\mathrm{B}$ and $\mathrm{C}$ ). The inferior rectus (IR in panel $\mathrm{E}$ ) as well as the inferior obliquus tendon (arrows in panel F) inserts to the sclera. A multi-layered sheath covers the sclera (panels $E$ and $F$ ) and some of them enclose the inferior obliquus (panel D). Panels A-D (or panels $E$ and F) were prepared at the same magnification (scale bars: $1 \mathrm{~mm}$ in panels $A$ and E). Other abbreviation — see Figure 1.

angle of the orbit: 1) a major muscle origin from the maxilla (see the subsection above) and 2) another origin from a fibrous tissue around the lacrimal sac. The latter tissue was still mesenchymal at midterm (Fig. 6C), but at late-term, muscle fibres appeared to intermingle with the vein-rich fibrous tissue around the lacrimal sac (Fig. 6F).

\section{DISCUSSION}

The adult 10 frequently shows anatomic variations (see the Introduction). According to the present observation of foetus sections, the fascial connection as well as a direct contact between the $I O$ and the inferior or lateral rectus seemed to provide variant muscular bridges as reported in adults. Moreover, the 


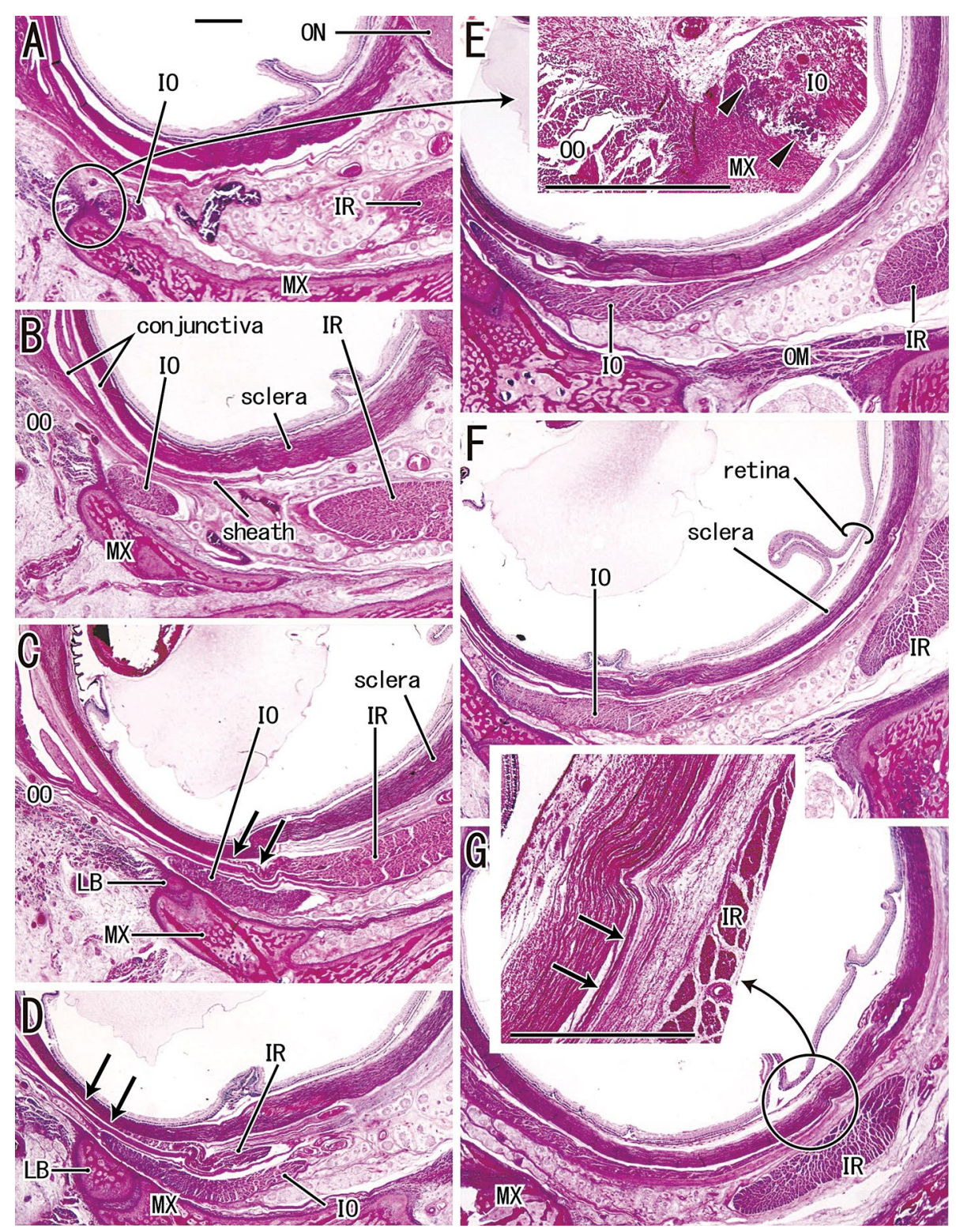

Figure 5. A-G. Inferior obliquus muscle in a late-term foetus of $276 \mathrm{~mm}$ crown-rump length. Haematoxylin and eosin staining. Sagittal sections. Panel $A$ displays the most medial plane in the figure. Inserts in panels $E$ and $G$ are higher magnification views of a circle in panels $A$ and $G$, respectively. Panel A shows a muscular origin of the inferior obliquus (IO; arrowheads in the insert), while panel $\mathrm{G}$ exhibits the insertion to the sclera (arrows in the insert). The inferior rectus (IR) inserts to the sclera (arrows in panels $C$ and $D$ ). Outside of the sclera, a thick sheath is seen connecting to the conjunctiva (panel $B$ ): it covers the inferior rectus in panels $C$ and $D$. Panels $A-G$ (or two inserts) were prepared at the same magnification (scale bars: $1 \mathrm{~mm}$ in panels $\mathrm{A}$ and the inserts). Other abbreviation — see Figure 1.

two attachment sites at the origin seemed to cause "tears" of muscle belly to provide double or multiple muscle bundles in adults. Therefore, in spite of the limited number, the present specimens contained seeds of any types of adult variations. In general, numerous variations have been reported in skeletal muscles. Although it is a small striated muscle without contribution to a joint movement, the 10 seemed to be the best sample for presentation of a hypothetical association between the foetal development and the adult variants.

At GA 7 weeks, the IO appeared to extend along the mediolateral axis: this direction was quite different from the extraocular recti extending along the anteroposterior axis. In spite of the early difference, the expanding eyeball made both of the recti and 10 take curved courses at GA 8 weeks. A tissue of the sclera was dense in the anterior part near the con- 

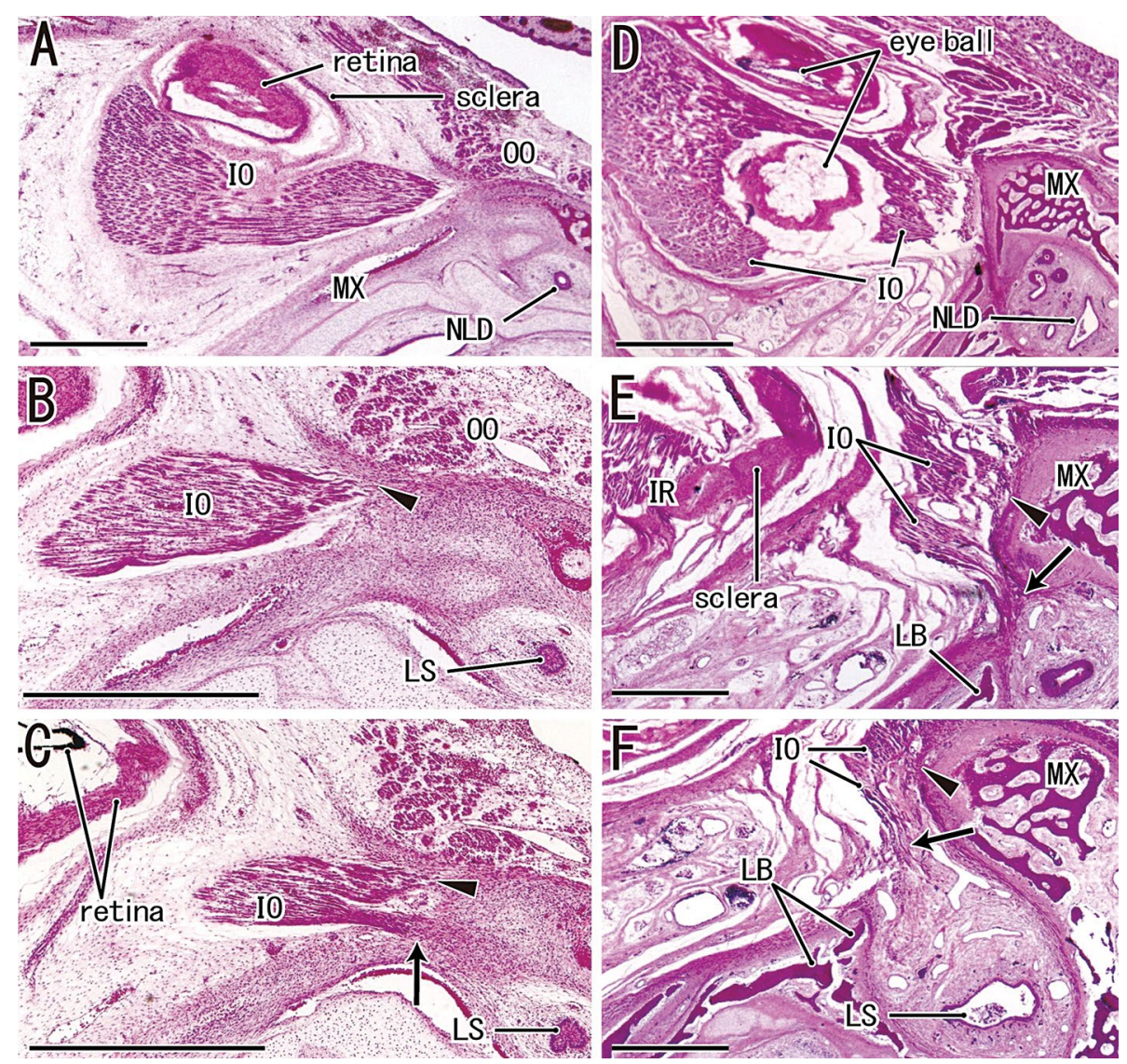

Figure 6. A-F. Double attachment sites at the origin of the inferior obliquus muscle at midterm and late-term. Haematoxylin and eosin staining. Horizontal sections. Panels $A-C$, a specimen of $118 \mathrm{~mm}$ crown-rump length (CRL); panels D-F, a specimen of $262 \mathrm{~mm}$ CRL. Panel A or D displays the most inferior site in each specimen. Arrowhead (panels $B, C, E, F$ ) indicates the muscle origin from the maxilla (MX), while arrow (panels C, E, F) another origin from a fibrous tissue around the lacrimal sac (LS). In panel D, the eye ball was cut at two sites because of an artefact bending with shrinkage after death. All scale bars, $1 \mathrm{~mm}$. Other abbreviation — see Figure 1.

junctiva and loose in the posterior part still at midterm. Thus, the muscle insertion established earlier in the recti than the IO. Therefore, a traction by the insertion seemed to play a little role for developing muscles wrapping around the eyeball. The expanding eyeball was most likely to directly push the muscle belly to be curved.

The 10 arose from two structures in foetuses: a major muscle bundle from the maxilla and 2) a minor part from a vein-rich fibrous tissue around the lacrimal sac. This dual origin seemed not to be known in adults $[5,11,15]$. Not the IO but the orbicularis oculi lacrimal part originates from the lacrimal sac according to these textbooks. We did not deny a possibility that the minor origin of the $1 \mathrm{O}$ would be degenerated after birth. However, at least in newborns, the 10 muscle fibres from the lacrimal sac might play a role for the lacrimal drainage.
The muscle insertion morphology in late-term foetuses was different from the adult one: not only the $\mathrm{IO}$ but the extraocular recti carried definite tendons in contrast to unclear tendons of the adult recti $[11,15]$. After birth, the rectus insertion would change to form the so-called extraocular muscle pulley system [4,9]. We demonstrated a multilayered sheath outside of the sclera at late-term: the thickest fascia to the conjunctiva was similar to the developing check ligament [10]. However, according to the connection to the inferior rectus, it seemed to correspond to the suspensory ligament of the eye [11].

\section{Acknowledgements}

This work was supported by Six Talent Peaks Project in Jiangsu Province (SZCY-001) and Wuxi Modern Industrial Development Funding (N20202008).

Conflict of interest: None declared 


\section{REFERENCES}

1. Deangelis DD, Kraft SP. The double-bellied inferior oblique muscle: clinical correlates. J AAPOS. 2001; 5(2): 76-81, doi: $10.1067 / \mathrm{mpa} .2001 .111780$, indexed in Pubmed: 11304813.

2. De Angelis D, Makar I, Kraft S. Anatomic variations of the inferior oblique muscle: a potential cause of failed inferior oblique weakening surgery. Am J Ophthalmol. 1999; 128(4): 485-488, doi: 10.1016/s0002-9394(99)00225-1.

3. de Haan $A B$, Willekens $B$, Klooster J, et al. The prenatal development of the human orbit. Strabismus. 2006; 14(1): 51-56, doi: 10.1080/09273970600579788, indexed in Pubmed: 16513570.

4. Demer JL, Miller JM, Poukens V, et al. Evidence for fibromuscular pulleys of the recti extraocular muscles. Invest Ophthalmol Vis Sci. 1995; 36(6): 1125-1136, indexed in Pubmed: 7730022.

5. Dutton JJ. Atlas of clinical and surgical orbital anatomy. WB Saunders, Philadelphia 2011.

6. Harayama K, Amemiya T, Nishimura H. Development of rectus muscles during fetal life: insertion sites and width. Invest Ophthalmol Vis Sci. 1980; 19(5): 468-474, indexed in Pubmed: 7372413.

7. Katori Y, Rodríguez-Vázquez JF, Kawase T, et al. Early fetal development of hard tissue pulleys for the human superior oblique and tensor veli palatini muscles. Ann Anat. 2011; 193(2): 127-133, doi: 10.1016/j.aanat.2011.01.004, indexed in Pubmed: 21334866.
8. Kocabiyik N. Orbital muscles. In Bergman's comprehensive encyclopedia of human anatomic variations. 1st ed. Wiley \& Sons 2016: 207-211.

9. Kono R, Poukens V, Demer JL. Quantitative analysis of the structure of the human extraocular muscle pulley system. Invest Ophthalmol Vis Sci. 2002; 43(9): 2923-2932, indexed in Pubmed: 12202511.

10. Osanai $\mathrm{H}$, Rodríguez-Vázquez JF, Abe $\mathrm{H}$, et al. Fetal check ligament connected between the conjunctiva and the medial and lateral recti. Invest Ophthalmol Vis Sci. 2011; 52(10): 7175-7179, doi: 10.1167/iovs.11-7426, indexed in Pubmed: 21791584.

11. Standling S. Gray's Anatomy, 39th ed. Elsevier Churchill Livingstone, London 2005: 684-694.

12. Tawfik HA, Dutton JJ. Embryologic and Fetal Development of the Human Orbit. Ophthalmic Plast Reconstr Surg. 2018; 34(5): 405-421, doi: 10.1097/IOP.0000000000001172, indexed in Pubmed: 30134385.

13. Yalçin B, Kocabiyik N, Ozan $\mathrm{H}$, et al. Muscular bridge between the inferior oblique and inferior rectus muscles. Am J Ophthalmol. 2004; 137(1): 121-124, doi: 10.1016/ s0002-9394(03)00787-6.

14. Yalçin $B$, Ozan $H$. Insertional pattern of the inferior oblique muscle. Am J Ophthalmol. 2005; 137: 121-124, doi: 10.1016/j.ajo.2004.10.057, indexed in Pubmed: 15767061.

15. Warwick R. Eugene Wolff's anatomy of the eye and orbit. 7th ed. WB Saunders, Philadelphia 1976. 